\title{
TOWARD EMPIRICAL EVALUATION OF LEFT VENTRICLE FUNCTION: A NOVEL MATHEMATICAL MAPPING
}

\author{
J.O. Dabiri, M. Gharib \\ Bioengineering Option, Califomia Institute of Technology, Pasadena, CA, USA
}

\begin{abstract}
A strategy is developed to facilitate quantitative analysis of left ventricle morphology based on clinically measured surface geometry and muscle fiber patterns rather than lower order geometric approximations previously required. A transfer function is derived which maps measured three-dimensional ventricle surfaces and associated muscle fiber patterns to a right circular cylinder, while preserving characteristic kinematics of the system. Functional analysis of ventricular morphology at various stages of the cardiac cycle proceeds by using classical methods on the cylindrical ventricle model, with substantially reduced analytical complexity when compared to similar calculations on the real ventricle shape. Functional morphology of the real ventricle shape at any stage of the cardiac cycle is subsequently deduced by applying the inverse of the transfer function in order to map the computed right circular cylinder back to its corresponding real ventricle shape. Limitations of the method are discussed in the context of real left ventricle performance, and extension of the method for analysis of functional morphology in other biomechanical systems is explored.

Keywords - ventricle, myocardium, mechanics, modeling
\end{abstract}

\section{INTRODUCTION}

Functional analysis of left ventricle morphology is aided by a model to correlate fiber orientation with observed ejection fraction. Reference [1] and subsequently others [2] have verified the existence of helically oriented fibers near the endocardial surface of the left ventricle. Similar fiber patterns have been observed in the epicardium [2]. It has been demonstrated that a helical fiber configuration is sufficient to achieve observed ventricular ejection fractions above 50 percent [3] given the limitation that papillary muscle typically shortens only 15 to 20 percent under physiological conditions [4]. By contrast, a system wholly composed of circular or constrictor fibers is inadequate.

Reference [5] first derived closed-form solutions of the ejection fraction in terms of parameters associated with a helical fiber configuration. However, this model and others subsequent to it (e.g. [6]) are typically confined to cylindrical, spherical, and ellipsoidal surfaces. Efforts to progress beyond these baseline geometries have encountered significant increases in computational effort and associated expense [5,7]. Indeed, calculations on geometries more closely approximating the real ventricle shape have not yet been accomplished.

This longstanding obstacle to more direct analysis of left ventricle function may be overcome by establishing a relationship between observed muscle fiber kinematics in the real ventricle and computed kinematics on a simpler geometry. This paper describes development of one such relationship and a strategic context for its implementation. A mathematical transfer function is constructed to broaden applicability of previous results on cylindrical geometries to clinically measured left ventricle shapes. Effectiveness of the method is based on preservation of a set of kinematic parameters within a mathematical mapping from the real ventricle shape to a right circular cylinder (and the corresponding inverse mapping). The generality of the mapping suggests it may find application not only in other cardiac subunits, but also more broadly, in analysis of other biomechanical systems for which morphology and function are intimately related. Limitations of the technique will be considered light of these potential applications.

\section{METHODOLOGY}

The strategy for quantitative analysis of real ventricular function using an idealized cylindrical system is as follows. A mathematical mapping function is desired which, when applied to the real ventricle geometry at some stage in the cardiac cycle (e.g. end diastole), will result in a right circular cylinder. This idealized cylindrical ventricle must be kinematically analogous to the real ventricle, where analogy is ascertained by metrics detailed in a later section. Using classical methods of analysis on the cylinder model $[5,6]$, its geometry at any other stage in the cardiac cycle (e.g. end systole) is determinable. The inverse of the original mathematical mapping function is then applied to this computed cylinder geometry to arrive at its corresponding real ventricle shape. Thus, given clinical measurements of the ventricle at a single stage of the cardiac cycle, the surface geometry and muscle fiber patterns of the ventricle at any other stage of the cardiac cycle may be ascertained. Fig. 1 illustrates the mapping protocol.

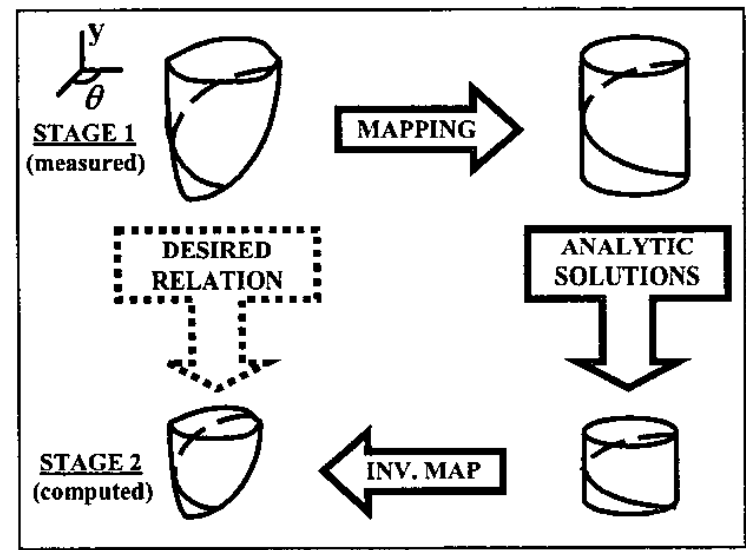

Fig. 1. Mapping protocol. Method proceeds clockwise from the clinically measured ventricle shape in the upper left to the computed ventricle shape in the lower left. Helix on lateral surface represents single muscle fiber. 


\section{ANALYSIS}

The analysis described here will proceed considering only the epicardial surface and its associated muscle fiber patterns. Extension to the endocardial surface and intermediate muscle matrix follows similarly.

To ensure that the ventricle shapes derived using this method are uniquely correct, certain requirements must be imposed on the mathematical mapping and its inverse (which, ipso facto, must exist). First, the mapping should be single-valued, lest we arrive at the physically impossible quandary of a multiple-valued solution to the kinematic problem. Second, the magnitude of body twisting and muscle fiber shortening between the two cardiac stages of interest must be the same for the idealized cylinders as it is for the real ventricle shapes. Since the shape of the ventricle at any stage of the cardiac cycle is uniquely determined by the current body twist and fiber length, this condition guarantees that the calculated solutions are not only physically feasible but also uniquely correct.

Uniqueness requires exactly two constraints for the two degrees of freedom in the mapping to a right circular cylinder (i.e. height and radius). These are

(i.) conservation of fiber length between each real ventricle geometry and its idealized cylindrical analog;

(ii.) conservation of body volume between each real ventricle and its idealized cylindrical analog.

Constraint (i.) conveniently satisfies the requirement of equal fiber shortening between the two cardiac stages for the real ventricle and its idealized cylindrical analog. The second constraint facilitates calculation of all transient volume-related parameters (e.g. ejection fraction) on the idealized cylinders rather than the more complex real ventricle geometries. Note that no constraint has been placed on the form of the ventricle body surface (e.g. ellipsoidal approximation) to preserve robustness of the technique. Indeed, calculations proceed using a general epicardial surface description in the form

$$
r_{\text {surf }} \equiv r(y, \theta) \text {. }
$$

Each helically oriented muscle fiber is given a similarly general description, with the angle $\theta$ described parametrically on the epicardial surface as

$$
\theta=(2 \pi f y) / H_{\text {vent }}
$$

In (2), $f$ is the number of times the muscle fiber circuits the epicardial surface (providing a measure of body twist between cardiac stages), $y$ is the vertical coordinate in a predefined orientation (see Fig. 1), and $H_{v e n t}$ is the height of the real ventricle in the same orientation.

\section{RESULTS}

According to the requirements set forth in the previous section, the idealized right circular cylinder (radius $R_{c y l}$, height $H_{c y l}$ ) corresponding to any given epicardial surface geometry and muscle fiber pattern described by (1) and (2) is computed by the mapping

$$
\begin{gathered}
R_{c y l}=\left\{\int_{0}^{H_{v e n t}}\left[\frac{2 \pi f r}{H_{v e n t}}+1\right]^{\frac{1}{2}} d y\right\}^{2}-H_{c y l}^{2} / 2 \pi f H_{c y l} \\
H_{c y l}=\int_{0}^{H_{v e n t}} \int_{0}^{2 \pi f} r^{2} d \theta d y / 2 \pi r_{c y l}^{2}
\end{gathered}
$$

Thus, the mapping consists of the nonlinear algebraic set (3) and (4) for the two cylinder parameters $R_{c y l}$ and $H_{c y l}$. Inversely, given these parameters, it is a straightforward exercise to determine the mapping to the epicardial surface and associated fiber patterns (1) and (2), respectively.

\section{DISCUSSION}

The strategy for this technique lies in a vein similar to the Joukowski and Schwarz-Christoffel transformations in fluid dynamics [8]. However, since this mapping is not conformal in general, we are free of both the need to specify a holomorphic transfer function and the requirement that holomorphic functions be used to describe the surfaces to be transformed. Accordingly, the limiting requirement becomes the ability to specify the surface geometry and fiber patterns in closed form. Given a set of surface coordinate points (e.g. from CT or MRI scan), various commercial software programs are available for that task.

\section{CONCLUSION}

The mapping technique developed here allows for the first time quantitative analysis of left ventricle kinematics based on clinical data rather than lower order approximations. When implemented computationally, the technique facilitates analysis of very large morphological data sets possessing high spatial and temporal resolution, making it compatible with clinical cardiac image acquisition tools. The generality of the technique may extend its application to functional analysis of other biomechanical systems where sufficient closed-form morphological descriptions are available.

\section{REFERENCES}

[1] D. Streeter et al., "Fiber orientation in the canine left ventricle," Circ. Res., vol. 24, pp. 339-347, 1969.

[2] F. Torrent-Guasp et al., "Spatial orientation of the ventricular muscle band," $J$. Thorac. Cardiov. Sur, vol. 122, pp. 389-392, 2001.

[3] J. Kennedy et al., "Quantitative angiocardiography I," Circulation, vol. 34, pp. 272-278, 1966.

[4] D. Brutsaert, E. Sonnenblick, "Force-velocity-length-time relations in beart muscle of the cat," Circ. Res., vol. 24, p. 137, 1969.

[5] E. Sallin, "Fiber orientation and ejection fraction in the human left ventricle," Biophys J., vol. 9, pp. 954-964, 1969.

[6] N. Ingels, "Myocardial fiber architecture and left ventricular function," Technol. Health Care, vol. 5, pp. 45-52, 1997.

[7] C. Peskin, D. McQueen, "Cardiac fluid dynamics," Crit. Rev. Biomed. Eng., vol. 20, pp. 451-459, 1992.

[8] L. Milne-Thompson, Theoretical Hydrodynamics, $5^{\text {th }}$ ed., London: Dover, 744 pp., 1968. 